Originalien

Orthopäde $2021 \cdot 50: 435-445$

https://doi.org/10.1007/s00132-020-04000-9

Online publiziert: 6. Oktober 2020

(c) Der/die Autor(en) 2020

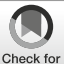

Hans-Rudolf Weiss ${ }^{1,2} \cdot$ Manuel Lay $^{3} \cdot$ Sarah Seibel $^{1} \cdot$ Alexander Kleban $^{4}$

'Gesundheitsforum Nahetal, Gensingen, Deutschland

${ }^{2}$ Neu Bamberg, Deutschland

${ }^{3}$ Orthopädie-Technik Lay, Zell-Barl, Deutschland

${ }^{4}$ Lomonosov Moscow State University, Moskau, Russland

\title{
Ist eine Verbesserung der Behandlungssicherheit in der Korsettversorgung von Skoliosepatienten durch Anwendung standardisierter CAD-Algorithmen möglich?
}

kuläre Skoliosen, Skoliosen bei mesenchymalen Defekten und vielen anderen Grunderkrankungen und Syndromen) [6]. Den Hauptteil aller Skoliosen stellen allerdings die Skoliosen mit unbekannter Ursache (idiopathische Skoliosen), von welchen die idiopathischen Adoleszentenskoliosen insgesamt mit ca. $80 \%$ am häufigsten auftreten. Daher gibt es zu dieser Skolioseform auch die meisten wissenschaftlichen Untersuchungen [1, $10,15]$.

Die idiopathische Adoleszentenskoliose tritt während des puberalen Wachstumsschubes auf und betrifft vorzugsweise Mädchen (weiblich zu männlich ca. 4:1), bei Krümmungen mit einem Krümmungswinkel von $40^{\circ}$ und mehr ist das Geschlechterverhältnis noch weiter zu Ungunsten der Mädchen verschoben (weiblich zu männlich ca. 10:1) [1, 10, 15].

Die Behandlung der idiopathischen Skoliose besteht aus:

- Verlaufsbeobachtung

- Physiotherapie

- Korsettversorgung

- Operation

Die Indikationsleitlinien zur konservativen Skoliosebehandlung sind erstmals 2006 veröffentlicht worden [33] und ha- ben sich seit dem nur unwesentlich geändert [41].

Im Hauptwachstumsschub kann die Progressionswahrscheinlichkeit nach Lonstein und Carlson für den Einzelfall errechnet werden [18]. Ab einer Progressionswahrscheinlichkeit von $60 \%$ besteht eine Korsettindikation [33, 41].

Die Korsettversorgung von Kindern und Adoleszenten kann heutzutage als evidenzbasierte Maßnahme angesehen werden [23, 30, 32]. Allerdings existieren weltweit eine Vielzahl unterschiedlicher Behandlungsansätze und Behandlungsphilosophien. Die Softbrace-Behandlung oder die reine Nachtversorgung im Hauptindikationsbereich $\left(25-40^{\circ}\right)$ haben sich im Grunde nicht bewährt [8, 11, 12, 21, 43]. Die Versorgung mit dem

\begin{tabular}{|ll}
\hline \multicolumn{2}{|l}{ Abkürzungen } \\
\hline$A T R$ & "Angle of trunk rotation“ \\
\hline BRAIST & $\begin{array}{l}\text { Bracing of Adolescent Idiopathic } \\
\text { Scoliosis Trial }\end{array}$ \\
\hline$C A D$ & "Computer aided design" \\
\hline$C A M$ & "Computer aided manufacturing" \\
\hline$G B W$ & Gensingen-Brace \\
\hline$S D$ & Standardabweichung \\
\hline$S R S$ & Scoliosis Research Society \\
\hline
\end{tabular}



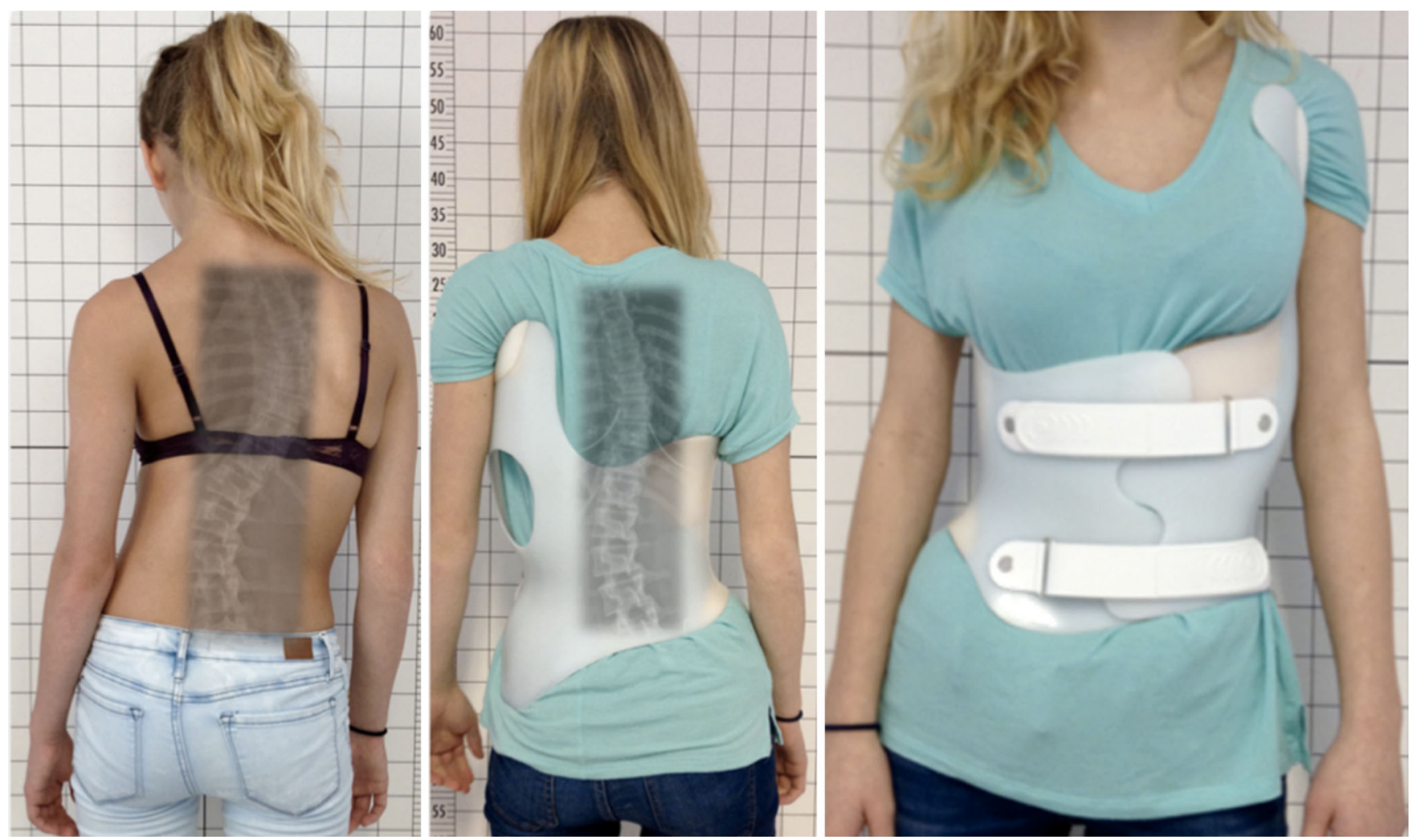

Abb. 1 × 13-Jährige (Risser 1) mit einem Krümmungswinkel thorakal von $53^{\circ}$, im Korsett korrigiert auf $26^{\circ} .3$ Monate nach Behandlungsabschluss betrug das Endergebnis $39^{\circ}$, der ATR (,angle of trunk rotation“) hatte sich von 18 auf $13^{\circ}$ verringert

Boston-Brace wurde durch eine randomisierte Studie belegt [30]. Allerdings hat sich in späteren Studien gezeigt, dass Chêneau-basierte Versorgungen zu besseren Endergebnissen führen können $[13,14,16,19,20,32,37,40]$. In einem aktuellen Review zur Korsettversorgung von Kindern und Jugendlichen fanden sich jedoch auch bei Chêneau-basierten Korsetten recht unterschiedliche Ergebnisse $[4,13,14,16,19,20,24,32,37,40$, 44]. Die Erfolgsquoten in den zitierten Studien schwankten zwischen 50 und $90 \%$ [42].

Dies mag zum einen daran liegen, dass immer noch viele Korsette nach Gipsabdruck hergestellt werden. Bei dieser Herstellungsweise ergeben sich kaum Möglichkeiten zur Standardisierung. Die Korsette werden für jeden Patienten individuell neu modelliert und somit kann man nicht auf bereits erfolgreich getesteten und standardisierten Modellen für unterschiedliche Krümmungsmuster aufbauen. CAD/CAM (,,computer aided design“/,,computer aided manufacturing“) kann zur Vereinheitlichung des Versorgungsstandards beitragen. Jedoch bringt nicht die Nutzung der CAD/CAMTechnologie alleine eine Verbesserung der Behandlungsergebnisse.

Dabei zeigen individuell hergestellte Korsette nicht per se schlechtere Ergebnisse als eine CAD-Versorgung. Allerdings können viele Orthopädietechniker wegen ihrer geringen Fallzahl nicht die zur sicheren Versorgung notwendige Expertise entwickeln.

Von Experten überwachte und ständig verbesserte CAD/CAM-Korsettbibliotheken lassen den Korsettstandard insgesamt verbessern. Entsprechende Versorgungen benötigen keine digitalen Nacharbeiten mehr. Bei solch standardisierten Systemen werden Korsettformen für unterschiedliche Krümmungsmuster seit mehr als einem Jahrzehnt weiterentwickelt und qualitätsgesicherte Grundmodelle von erfahrenen CADDesignern bereits digital and den 3DScan des Patienten angepasst, sodass wesentliche Veränderungen durch den Techniker vor Ort nicht vonnöten sind. Die Fähigkeiten und Fertigkeiten zur Feinanpassung entsprechender Modelle an die Patienten werden in der Re- gel innerhalb weniger Tage in speziell ausgeschriebenen Kursen vermittelt.

In Deutschland ist das Rigo-ChêneauKorsett und das Regnier-Chêneau-Korsett gut eingeführt. International hat das Gensingen-Brace (GBW) eine recht weite Verbreitung gefunden. Das GBW wurde 2010 eingeführt [35] und wird in Kooperation mit internationalen Spezialisten stetig weiterentwickelt. Zur Versorgung mit dem GWB wurde die auch von Dr. Chêneau verwendete Lehnert-SchrothKlassifikation (funktionell dreibogig/ funktionell vierbogig) zur augmentierten Lehnert-Schroth-Klassifikation mit 7 Grundmustern erweitert [35, 40].

Im Folgenden sind die Grundmuster der augmentierten Lehnert-SchrothKlassifikation aufgeführt:

- 3BH (großbogige Thorakalkrümmung ohne wesentliche Lumbalkrümmung/Lenke-A-Muster)

- 3BTL (großbogige Thorakalkrümmung ohne wesentliche Lumbalkrümmung/Lenke-A-Muster mit Scheitelwirbel Th 12) 
- 3BN (thorakale Hauptkrümmung mit kleinerer und kurzbogiger Lumbalkrümmung/Lenke B)

- 3BL (thorakale Hauptkrümmung mit kleinerer und langbogiger Lumbalkrümmung/Lenke C)

- 4B (Double-major-Muster mit struktureller Thorakal- und Lumbalkrümmung/Lenke 4C/Lenke 6)

- 4BL (lumbale Hauptkrümmung mit kleinerer und kurzbogiger Thorakalkrümmung/Lenke 5)

- 4BTL (thorakolumbale Hauptkrümmung mit kleinerer und kurzbogiger Thorakalkrümmung/Lenke 5, Scheitelwirbel L1)

Die der GBW-Versorgung (• Abb. 1) zugrunde liegenden CAD-Algorithmen werden für jedes der 7 Grundmuster festgelegt. Die CAD-Korrekturparameter für die einzelnen Krümmungsabschnitte werden für unterschiedliche Krümmungsstärken $\quad\left(20-30^{\circ} ; 30-40^{\circ}\right.$; $40-50^{\circ}$ und für Einzelfälle auch darüber hinaus) und unterschiedliche Reifegrade individuell eingestellt. Im Folgenden sollen die in unserer Abteilung mit dem GBW erzielten Ergebnisse dargestellt und mit den Ergebnissen des BostonKorsetts [30] hinsichtlich der Erfolgsrate („rate of success“) als Zielparameter verglichen werden.

\section{Material und Methode}

Am Stichtag (08.12.2019) wurde die prospektiv angelegte Datenbank unserer Abteilung retrospektiv ausgewertet. Es handelt sich um eine retrospektive Untersuchung (Chart Review) derjenigen Patientinnen, welche die unten aufgeführten Einschlusskriterien erfüllten. Diese Einschlusskriterien für Korsettstudien bei Skoliosen gehen auf Empfehlungen der Scoliosis Research Society (SRS) zurück [25].

Einschlusskriterien zur Aufnahme in die seit 2011 geführte Datenbank waren:

- ausschließlich Mädchen

- Diagnose einer idiopathischen Adoleszentenskoliose

- Alter 10-14 Jahre (bei Erstbeobachtung)

- Risser 0-2

- Krümmungswinkel ab $25^{\circ} \mathrm{Cobb}$

Am Stichtag befanden sich 151 Patientinnen in unserer Datenbank. Ausgewertet wurden folgende Patientinnengruppen:

- Patientinnen mit einem minimalen Follow-up von 18 Monaten (Gruppe 1)

- Endresultate insgesamt (Gruppe II)

- Endresultate mit einem Cobb-Winkel von $25-40^{\circ}$ (SRS-Einschlusskriterien/ Gruppe III)

- Endresultate mit einem Cobb-Winkel von $40^{\circ}$ und darüber (Gruppe IV)
Die Gruppe I ist die Gesamtgruppe aller in diese Untersuchung einbezogener Patientinnen. Diese beinhaltet die Gruppen II-IV als Untergruppen, welche wir einer stärker differenzierten Betrachtung unterziehen wollen.

Die Endresultate wurden 3-36 Monate nach vollständiger Korsettabschulung ermittelt. Die Patientinnen mit einem Krümmungswinkel von mehr als $40^{\circ}$ hatten sich ausnahmslos gegen eine Operation und meist für eine Weiterbehandlung in unserer Abteilung entschieden, da in der vorbehandelnden Praxis/Klinik nach stellen der Operationsindikation eine Behandlung mit einem Korsett abgelehnt worden war.

Die 87 Patientinnen der Gruppe I mit einem durchschnittlichen CobbWinkel von $40,9^{\circ}$ (SD 12,7) hatten einen Beobachtungszeitraum von mindestens 18 Monaten. Das Durchschnittsalter in dieser Gruppe betrug 12,3 Jahre (SD $0,96)$, die Menarche war durchschnittlich seit 2,1 Monaten aufgetreten (SD 3,6). Das durchschnittliche Risser-Stadium lag bei 0,6 (SD 0,9).

$49 \%$ der Patientinnen hatten eine thorakale Hauptkrümmung, $35 \%$ eine Double-major-Krümmung, $8 \%$ eine lumbale und weitere $8 \%$ eine thorakolumbale Hauptkrümmung. Die durchschnittliche Korsetttragezeit wurde mit 20,6 h/Tag (SD 2,9) angegeben. Die Angaben hier$\mathrm{zu}$ stammen von den Patientinnen und deren Eltern.

Hier steht eine Anzeige. 
Der durchschnittliche Beobachtungszeitraum aller in dieser Studie untersuchten Patientinnen (Gruppe I) betrug 30,8 Monate (SD 12,3). Der durchschnittliche Beobachtungszeitraum aller in dieser Studie abgeschlossenen Patientinnen (Gruppe II) betrug 36,8 Monate (SD 14,9).

Die Reifecharakteristika und die Cobb-Winkel-Verteilung aller 4 Gruppen sind der $\bullet$ Tab. 1 und 2 zu entnehmen. Die Krümmungsmusterverteilung innerhalb der 4 Gruppen ist in der - Tab. 3 aufgeführt, wobei die Gruppe I die Gruppen mit den Endresultaten (Gruppen II-IV) beinhaltet.

Die Progressionsrate (Verschlechterung des Cobb-Winkels $6^{\circ}$ und mehr) wurde für alle 4 Gruppen ermittelt, ebenso wie der Prozentsatz der stabilisierten $\left( \pm 5^{\circ}\right)$ und verbesserten Patientinnen $\left(6^{\circ}\right.$ und mehr). Die ermittelte Erfolgsrate (stabilisierte + verbesserte Hauptkrümmungen) als Zielparameter unserer Untersuchung wurde mit der Erfolgsrate der BRAIST(Bracing of Adolescent Idiopathic Scoliosis Trial)-Studie von Weinstein und Mitarbeitern [30] verglichen.

\section{Gensingen-Brace (GBW)}

Das GBW ist ein weiterentwickeltes Chêneau-Korsett mit individuellem Design, welches Computer unterstützt (CAD) angepasst werden kann [37, 39]. Die einzelnen Produktionsschritte sind bereits in der Literatur beschrieben [39].

Zunächst wird die Patientin gescannt und es werden die Patientendaten erhoben und gemeinsam mit dem Röntgenbild in die Datenbank eingepflegt. Anhand dieser Daten wird zunächst einmal das dem Krümmungsmuster entsprechende Grundmodell aus der Korsettbibliothek ausgewählt.

Der Patientenscan wird beschnitten und skaliert. Anschließend wird das ausgewählte Korsett in die Szene eingefügt und entsprechend der individuellen Körperform angepasst. Danach werden entsprechend die für das jeweilige Muster und die jeweilige Krümmungsstärke (Cobb-Winkel) festgelegten Korrekturalgorithmen angewendet. Im Ergebnis erhält man ein Korsettmodell, welches

Orthopäde 2021 · 50:435-445 https://doi.org/10.1007/s00132-020-04000-9

(c) Der/die Autor(en) 2020

H.-R. Weiss · M. Lay · S. Seibel · A. Kleban

\section{Ist eine Verbesserung der Behandlungssicherheit in der Korsettversorgung von Skoliosepatienten durch Anwendung standardisierter CAD-Algorithmen möglich?}

\section{Zusammenfassung}

Hintergrund. Die Versorgung von Skoliosepatienten mit korrigierenden Rumpforthesen führt auch heutzutage noch zu recht unterschiedlichen Ergebnissen. Publizierte Erfolgsquoten zwischen 50 und $90 \%$ führen zwangsläufig zu der Frage, wie sich die Erfolgsquoten der Korsettversorgung steigern und vereinheitlichen lassen. Die Ergebnisse einer mit dieser Zielsetzung weiterentwickelten computerunterstützen (CAD/„Computer Aided Design“) ChêneauVersorgung werden dargestellt.

Methodik. Am Stichtag (08.12.2019) wurde die prospektiv angelegte Datenbank unserer Abteilung retrospektiv ausgewertet. Es waren ausschließlich unreife Mädchen mit einer Adoleszentenskoliose, Alter 10-14 Jahre, Risser 0-2 in die Datenbank aufgenommen worden.

Ergebnisse. Sowohl die Gesamtgruppe mit einem Beobachtungszeitraum von mindestens 18 Monaten als auch die
Patientinnengruppen mit bereits erreichtem Behandlungsabschluss zeigten Erfolgsraten zwischen 86 und $88 \%$. Die Ergebnisse insgesamt waren signifikant besser als die Erfolgsrate der Boston-Brace-Kontrollgruppe (BRAIST) von $72 \%$. Auch im Vergleich mit den Ergebnissen anderer Chêneau-Derivate war die Erfolgsrate unserer Serie teils deutlich besser.

Schlussfolgerungen. Die Behandlungssicherheit für die Patienten mit Skoliosen sollte verbessert werden. Ein Ansatz hierzu kann die Verwendung standardisierter CADBibliotheken sein. Weitere Untersuchungen mit Studiendesigns höherer Evidenz sind notwendig, um die in unserer Untersuchung gefundenen Ergebnisse zu untermauern.

Schlüsselwörter

Rumpforthesen · Mädchen · Wachstum . Computer-aided design · Computer-aided manufacturing

\section{Is it possible to improve treatment safety in the brace treatment of scoliosis patients by using standardized CAD algorithms?}

\section{Abstract}

Background. The treatment of scoliosis patients with corrective braces today still leads to quite different results. The published success rates between 50 and $90 \%$ inevitably lead to the question of how the success rates of brace treatment can be increased and standardized. The results of a computer aided design (CAD) Chêneau application, developed with this objective, are presented.

Materials and methods. On the reporting date (December 8, 2019), the prospectively created database of our department was evaluated retrospectively. Only immature girls with adolescent idiopathic scoliosis (AIS), aged 10-14 years, Risser signs $0-2$, were included in the database.

Results. Both the entire group with an observation period of at least 18 months and the patient groups with completed treatment had success rates between 86 and $88 \%$. Overall, the results were significantly better than the $72 \%$ success rate of the Boston Brace Control Group (BRAIST). In comparison with the results of other Chêneau derivatives, the success rate of our series was distinctly better. Conclusions. Treatment safety in brace treatment for patients with scoliosis should be improved. One approach may be the use of standardized CAD libraries. Further investigations with study designs of higher evidence are necessary to support the results found in our investigation.

Keywords

Trunk orthoses · Girls · Growth · Computer-aided design - Computer-aided manufacturing 


\begin{tabular}{|c|c|c|c|c|}
\hline & $n$ & Alter & Menarche & Risser \\
\hline Gruppe I & 87 & 12,3 (SD 0,96) & $2,1(\mathrm{SD} 3,6)$ & $0,6(S D 0,9)$ \\
\hline Gruppe II & 39 & 12,4 (SD 0,99) & $2,5(\mathrm{SD} 3,8)$ & $0,7(S D 0,9)$ \\
\hline Gruppe III & 24 & $12,5(S D 0,98)$ & $3,0(S D 4,3)$ & $0,8(S D 0,9)$ \\
\hline Gruppe IV & 17 & 12,3 (SD 1,05) & $2,5(S D 3,4)$ & 0,8 (SD 1) \\
\hline
\end{tabular}

\begin{tabular}{|c|c|c|c|c|}
\hline & $n$ & Cobb Beginn & Cobb Korsett & Cobb Ende \\
\hline Gruppe I & 87 & 40,9 (SD 12,7) & $21,8(S D 13,1)$ & 38,2 (SD 16,2) \\
\hline Gruppe II & 39 & 37,9 (SD 10,8) & 19,6 (SD 11) & 34,5 (SD 14,5) \\
\hline Gruppe III & 24 & $30,8(S D 4,7)$ & $14,8(S D 7,9)$ & 27,3 (SD 8,8) \\
\hline Gruppe IV & 17 & $48,2(S D 7,4)$ & 25,3 (SD 12,3) & 44,8 (SD 14,2) \\
\hline
\end{tabular}

Tab. 3 Krümmungsmusterverteilung innerhalb der einzelnen Behandlungsgruppen

\begin{tabular}{|c|c|c|c|c|c|}
\hline & $n$ & $\begin{array}{l}\text { Thorakal } \\
\text { (in \%) }\end{array}$ & $\begin{array}{l}\text { Double major } \\
\text { (in \%) }\end{array}$ & $\begin{array}{l}\text { Lumbal } \\
\text { (in \%) }\end{array}$ & $\begin{array}{l}\text { Thorakolumbal } \\
\text { (in \%) }\end{array}$ \\
\hline Gruppe I & 87 & 49 & 35 & 8 & 8 \\
\hline Gruppe II & 39 & 41 & 36 & 13 & 10 \\
\hline Gruppe III & 24 & 42 & 29 & 16 & 13 \\
\hline Gruppe IV & 17 & 47 & 41 & 6 & 6 \\
\hline
\end{tabular}

das jeweilige Krümmungsmuster widerspiegelt (•Abb. 2).

\section{Kontrollgruppe}

Die randomisierte BRAIST-Untersuchung von Weinstein und Mitarbeitern [30] hat an die SRS-Kriterien für Korsettstudien [25] angelehnte Einschlusskriterien, wodurch Patientinnen definierter Reife und definierter Krümmungswinkel einbezogen werden, was die Vergleichbarkeit erleichtert. Ferner sind die Ergebnisse zum Boston-Korsett relativ homogen (Erfolgsraten um die $70 \%$ sowohl in der SRS-Multicenter-Studie von Nachemson und Petersen [23], als auch bei BRAIST [30]). Demgegenüber liegen die Erfolgsraten bei Chêneau-Versorgungen zwischen 50 und $90 \%$ [42], was einerseits die Auswahl einer geeigneten Studie erschwert. Andererseits folgen die meisten Studien zur Chêneau-Versorgung nicht den Einschlusskriterien der SRS und sind dadurch kaum vergleichbar. Daher haben wir uns für BRAIST [30] als Kontrollgruppe entschieden.

\section{Statistische Methoden}

Zum statistischen Vergleich unserer Ergebnisse mit der BRAIST-Studie wurde der von Goldberg und Mitarbeitern vorgeschlagene $\mathrm{z}$-Test zum Vergleich unterschiedlicher Proportionen angewen$\operatorname{det}[7,9]$. Gruppengrößen ab $n=30$ werden für diesen Test als optimal angesehen, allerdings werden kleinere Gruppen nicht als Ausschlusskriterium für diesen Test angesehen [7], zumal $n$ als zweiter Parameter neben der Stichprobenproportion (\%) das Signifikanzniveau des Ergebnisses direkt beeinflusst.

Zusätzlich wurde auch der Winkel der Rumpfrotation [5] (,angle of trunk rotation" [ATR]) zu Beginn und am Ende der Behandlung mithilfe des Skoliometers nach Bunnel für den Thorakalbereich als auch für den Lumbalbereich ermittelt. Die Veränderungen dieser Winkel wurden mithilfe des t-Tests auf statistische Signifikanz geprüft.

Die Ergebnisse der untersuchten Patientinnengruppen wurden zuvor auf Normalverteilung untersucht (ShapiroWilk-Test/alpha $=0,05$ ).

Der technische Fehler (Variabilität der Messungen) der Cobb-Winkel-Messung liegt zwischen 3 [28] und $6^{\circ}$ [17]. Daher nimmt man in wissenschaftlichen Untersuchungen erst $\mathrm{ab}$ einer Winkeländerung von $6^{\circ}$ und mehr eine tatsächliche Änderung an. Messunterschiede von $\pm 5^{\circ}$ werden als unverändert bewertet.

Die Messung des ATR mit dem Skoliometer hat eine hohe Reliabilität [22]. Der durchschnittliche technische Fehler liegt bei etwa $1^{\circ}[22,31]$.

\section{Ergebnisse}

bei Behandlungsabschluss. Daher haben wir alternativ für die vergleichbare Gruppe III aus unserer Untersuchung auch die Ergebnisse für dieses Erfolgskriterium ermittelt.

Da es sich bei der vorliegenden Untersuchung um ein Chart Review handelt, fand zur Auswertung der Ergebnisse keine Verblindung statt. Die Messungen von Cobb-Winkel und Rumpfrotation erfolgten ausschließlich durch den Erstautor.

Die Veränderungen des durchschnittlichen Cobb-Winkels (vor Behandlung/im Korsett/bei Behandlungsabschluss) sind für alle 4 Gruppen in $\bullet$ Tab. 2 dargestellt.

Die Korrektureffekte im Korsett liegen je nach Gruppe zwischen 47 und 52\% des Ausgangswertes und waren im t-Test in allen Gruppen hochsignifikant.

Die Erfolgsraten lagen zwischen 86 und $88 \%$, bei Anwendung der Erfolgskriterien der BRAIST-Studie [17] in der vergleichbaren Gruppe III bei $96 \%$ (• Tab. 4). Die Ergebnisse sind für die 


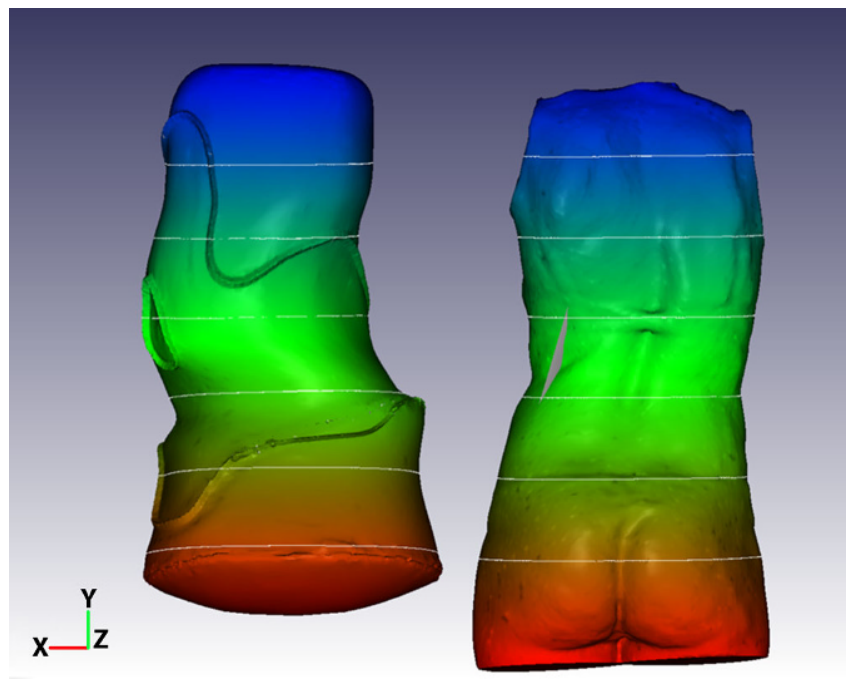

Abb. $\mathbf{2} \triangleleft \mathrm{CAD}$ (",computer aided design")-Szene mit Patientenscan (rechts) und modelliertem Korsett (links). Man erkennt, dass das Korsett das Krümmungsmuster spiegelt
Gruppen I-IV in der • Tab. 5 aufgegliedert.

Die Ergebnisse der statistischen Auswertung sind in - Tab. $6 \mathrm{zu}$ finden.

Im Vergleich zur BRAIST-Studie [30] sind die Erfolgsraten unserer Studie in den Gruppen I-III signifikant besser. Die Erfolgsquote der Gruppe IV unterscheidet sich nicht wesentlich von denen der Gruppen I-III. Die fehlende Signifikanz ist hier auf die geringe Gruppengröße zurückzuführen $(n=17)$.

Der thorakale ATR verringerte sich in der Gesamtgruppe (Gruppe I) von 10,5 auf $8,6^{\circ}(p<0,05)$, der lumbale von 6,4 auf $3,7^{\circ}(p>0,01)$.

\section{Diskussion}

Die BRAIST-Studie [30] hat relativ lockere Erfolgskriterien. Wenn eine Krümmung die $50^{\circ}$-Grenze nicht erreichte oder übertraf, wurde der Verlauf als erfolgreich bezeichnet. Dies bedeutet, dass diese Studie eine nicht näher bezeichnete Anzahl von Patienten beinhaltete, welche sich zwar um $6^{\circ}$ und mehr verschlechtert, die $50^{\circ}$-Grenze jedoch nicht erreicht hatten. Dementsprechend kann man davon ausgehen, dass sich bei Berücksichtigung unserer Erfolgskriterien (keine Verschlechterung von $6^{\circ}$ und mehr) in der BRAIST-Studie [30] eine weit geringere Erfolgsrate ergeben würde. Allerdings gibt es ansonsten keine andere Vergleichsgruppe aus einer Studie hoher Evidenz, welche sich an den SRS-Kriterien [25] für Stu- dien zur Korsettversorgung orientieren würde (Diagnose einer idiopathischen Adoleszentenskoliose, Alter 10-14 Jahre, Risser 0-2, Cobb-Winkel 25-40 ${ }^{\circ}$ ). Da in der BRAIST-Studie [30] auch Jungen eingeschlossen waren, wurde entsprechend der späteren Ausreifung die Altersspanne auf 10-15 Jahre erweitert.

Interessanterweise sind nicht nur die Ergebnisse der Gruppen I-III signifikant besser als die Ergebnisse der BRAISTStudie [30] mit den lockereren Erfolgskriterien, auch die Gruppe mit Krümmungen von $40^{\circ}$ und mehr lag im Ergebnis mit $88 \%$ deutlich besser als die BRAISTStudie $\left(20-40^{\circ}\right)$ [30], obwohl Krümmungen zwischen 40 und $64^{\circ}$ in der Gruppe enthalten waren.

Auch der Winkel der Rumpfrotation (ATR) hat sich in der Gesamtgruppe (Gruppe I) signifikant verbessert. Dies bedeutet, dass sich auch die kosmetisch bedeutsamen Parameter mit dem GBW verbessern lassen. Für die Patientinnen mag dies wichtiger sein als die Veränderungen des Cobb-Winkels, der sich am Ende statistisch nicht signifikant verbessert hat.

Die Ergebnisse unserer Untersuchung sind für die einzelnen Untergruppen, selbst unter Einschluss der Gruppe IV mit Krümmungen über $40^{\circ}$, recht homogen. Dies kann wohl auf die standardisierte CAD/CAM-Versorgung zurückgeführt werden. In Anbetracht dieser überdurchschnittlichen Ergebnisse sind Erfolgsraten von weniger als $70 \%$, wie bei einigen Studien mit dem
Chêneau-Korsett [4, 27, 44] oder mit Nachtorthesen [8], aus Sicht der Betroffenen nicht akzeptabel. Allerdings sind die Erfolgsraten in unterschiedlichen Studien zur Chêneau-Versorgung sehr variabel und liegen zwischen 50 und $90 \%[4,13,14,16,19,20,24,32,37$, 40, 44], wobei lediglich die Ergebnisse der Studie von Korovessis et al. [14] mit denen unserer Untersuchung vergleichbar waren. $\mathrm{Zu}$ komplex ist die optimale Planung und Modellierung einer Chêneau-Korsettversorgung, als dass sie von jedem Techniker und für jedes mögliche Krümmungsmuster nach einem kurzen Kurs leicht beherrscht werden könnte. Zudem ist durch die relativ geringe Prävalenz der behandlungsbedürftigen Skoliosen (ca. 0,5\%) in der Gesamtbevölkerung [1, 10, 15] die Möglichkeit begrenzt, in kürzerer Zeit Erfahrungen zu sammeln und die eigenen Fähigkeiten stetig zu verbessern.

Die Betroffenen erleiden durch jedwede Korsettversorgung mehr oder weniger bedeutsame Beeinträchtigungen der Lebensqualität. Diese Beeinträchtigung muss von einer bestmöglichen Erfolgsaussicht begleitet werden.

Daher sollte die Korsettversorgung von Patienten mit Wirbelsäulendeformitäten nur standardisierten und qualitätsgesicherten CAD/CAM-Versorgungen oder sehr erfahrenen Orthopädietechnikern mit einer entsprechen hohen Fallzahl und nachgewiesener Ergebnisqualität vorbehalten bleiben. Individuelle, teils wenig erfolgversprechende Versorgungen rechtfertigen den zeitlichen Einsatz und die Beeinträchtigungen der Betroffenen nicht.

Die Studien zur Korsettversorgung bei Skoliosen fokussieren fast alle ausschließlich aufden Cobb-Winkel, obwohl dieser für die betroffenen Patienten mit idiopathischer Adoleszentenskoliose nur von untergeordneter Bedeutung ist. In dieser zahlenmäßig größten Patientengruppe mit Skoliosen sind schwerwiegende Gesundheitsbeeinträchtigungen selbst bei unbehandelten Patienten die Ausnahme [1, 29, 38]. Daher sollte auch - oder gar in erster Linie - untersucht werden, welche Korsette in der Lage sind, die Rumpfasymmetrie positiv zu beeinflussen. Ein Parameter zur Mes- 
Tab. 4 Korrektureffekt im Korsett und Erfolgsrate

\begin{tabular}{llllll}
\hline & n & Korrektureffekt (in \%) & $\boldsymbol{p}$ & Erfolgsrate (in \%) \\
\hline Gruppe I & 87 & 47 & $<0,01$ & 86 \\
\hline Gruppe II & 39 & 48 & $<0,01$ & 87 \\
\hline Gruppe III & 24 & 52 & $<0,01$ & $88 / 96^{\mathrm{a}}$ \\
\hline Gruppe IV & 17 & 48 & $<0,01$ & 88 \\
\hline BRAIST [30] & 146 & Keine Angabe & - & 72
\end{tabular}

Die Korrektureffekte in der CAD-Chêneau-Orthese waren im t-Test hochsignifikant, ${ }^{a}$ bei Anwendung der Erfolgsparameter der BRAIST(Bracing of Adolescent Idiopathic Scoliosis Trial)-Studie (am Ende $\left.<50^{\circ}\right)$

Tab. 5 Resultate der Korsettversorgung innerhalb der einzelnen Gruppen

\begin{tabular}{l|llll} 
& $\boldsymbol{n}$ & Verschlechtert & Gleich & Verbessert \\
\hline Gruppe I & 87 & $12(14 \%)$ & $42(48 \%)$ & $33(38 \%)$ \\
Gruppe II & 39 & $5(13 \%)$ & $18(46 \%)$ & $16(41 \%)$ \\
Gruppe III & 24 & $3(13 \%)$ & $11(46 \%)$ & $10(42 \%)$ \\
Gruppe IV & 17 & $2(12 \%)$ & $7(41 \%)$ & $8(47 \%)$
\end{tabular}

Für jede Rubrik wird die Anzahl der Patientinnen und die Prozentwerte angegeben

\section{Tab. 6 Erfolgsquote der Korsettversorgung im Vergleich zu BRAIST [30]}

\begin{tabular}{|c|c|c|c|c|}
\hline & $n$ & Erfolgsquote (in \%) & z-Wert & $p$ \\
\hline Gruppe I & 87 & 86 & 2,58 & 0,01 \\
\hline Gruppe II & 39 & 87 & 1,96 & 0,05 \\
\hline Gruppe III & 24 & 88 & 1,96 & 0,05 \\
\hline Gruppe III ${ }^{\mathrm{a}}$ & 24 & 96 & 2,58 & 0,01 \\
\hline Gruppe IV & 17 & 88 & Ns & Ns \\
\hline BRAIST [30] & 146 & 72 & - & - \\
\hline
\end{tabular}

Tab. 7 Verteilung der progredienten Fälle je Risser-Stadium ${ }^{a}$

\begin{tabular}{|l|l|l|} 
& n & Davon progredient \\
\hline Risser 0 & 22 & $n=3$ \\
\hline Risser 1 & 5 & $n=1$ \\
\hline Risser 2 & 12 & $n=1$ \\
\hline${ }^{\text {a} A b g e s c h l o s s e n e ~ F a ̈ l l e ~(G r u p p e ~ I I) ~}$ \\
\hline
\end{tabular}

sung der Rumpfasymmetrie ist der ATR, gemessen mit dem Skoliometer nach Bunnel [5]. Allerdings beschreibt dieser Wert lediglich die Rumpfasymmetrie bei vorgebeugtem Rumpf. Auch wenn sich dieser Wert nur wenig verbessert, kann die Verbesserung der Rumpfasymmetrie im aufrechten Stand sehr deutlich ausfallen (• Abb. 3). Es gibt sogar Fälle ohne wesentliche Verbesserung des CobbWinkels, aber mit sehr bedeutsamen Verbesserungen der Rumpfasymmetrie (• Abb. 4; [36]). Grade für Patienten mit idiopathischer Adoleszentenskolio- se sollte mehr Wert auf Studien gelegt werden, welche auf die kosmetisch bedeutsamen klinischen Parameter fokussieren. Hilfreich hierfür könnte auch die Oberflächenvermessung sein bei Auswertung der bildhaften Darstellung sowie der ausgegebenen Messparameter (• Abb. 5).

Die Patientinnen der Gruppe I hatten eine minimale Beobachtungszeit von 18 Monaten, in dieser übergeordneten Gruppe sind allerdings auch die Patientinnen der Gruppen II-IV mit Behandlungsabschluss enthalten. Natürlich kann man für die noch nicht abgeschlossenen Patientinnen keine abschließende Beurteilung treffen. Betrachtet man allerdings die Durchschnittspatientin aus dieser Gruppe, so befindet sich diese $\mathrm{Pa}$ tientin zu Behandlungsbeginn noch im Hochrisikobereich des Wachstumsschubes ([1, 10, 15, 18]; • Tab. 1), während dieselbe Patientin nach mehr als 18 Mo- naten nur noch ein geringes Restwachstum aufweist und eine Krümmungsprogression damit weniger wahrscheinlich ist (-Abb. 6). Berücksichtigt man zusätzlich, dass die Durchschnittspatientin nach Lonstein und Carlson [18] einen Progressionsfaktor von mehr als 3 und somit eine Progressionswahrscheinlichkeit von nahezu $100 \%$ hat, so kann man schon den Ergebnissen der noch nicht abgeschlossenen Patientinnen nach einem Follow-up von mehr als 18 Monaten eine gewisse Evidenz zubilligen.

Ist man früher grundsätzlich davon ausgegangen, dass bis 2 Jahre nach Behandlungsabschluss eine zuvor erreichte Korrektur wieder zurückfällt, rechnet man heute bei Anwendung hochkorrigierender Rumpforthesen nicht mehr mit einer signifikanten Verschlechterung des Endergebnisses im Langzeitverlauf. Aulisa und Mitarbeiter haben mehr als 10 Jahre nach Korsettabschulung bleibende Korrekturen nachgewiesen, die sich nicht wesentlich von den Ergebnissen direkt nach Abschulung unterschieden [2].

Interessant ist auch, dass der Beobachtungszeitraum in der BRAIST-Studie [30] mit 24,2 Monaten deutlich kürzer war, als der unserer abgeschulten Gruppe II (36,8 Monate). Dies ist wohl einerseits darauf zurückzuführen, dass die Reduzierung der Tragezeit am Ende der Wachstumsphase in unserem Kollektiv in aller Regel über 18 Monate erfolgte, andererseits darauf, dass wir die Reduzierung der Tragezeit bei Ausgangswinkeln jenseits der $40^{\circ}$ Grenze regelmäßig auf etwa 24 Monate ausdehnen. Das in der Kontrollgruppe [30] verwendete BostonBrace wird in aller Regel über einen kürzeren Zeitraum abgeschult.

In einigen Studien findet man die Beobachtung, dass die reiferen Patienten, also die mit ohnehin geringerem Risiko, die geringste Progressionsneigung haben [27, 42]. Diese Beobachtung kann in Studien mit hochkorrigierenden Korsetten nicht bestätigt werden [2, 3]. Die Anzahl der progredienten Fälle in unserer Untersuchung ist zu gering, um eine Korrelation mit dem Risser-Stadium zu erstellen. Die progredienten Fälle der Gruppe II (abgeschlossenen Behandlungsfälle) fin- 


\section{Originalien}
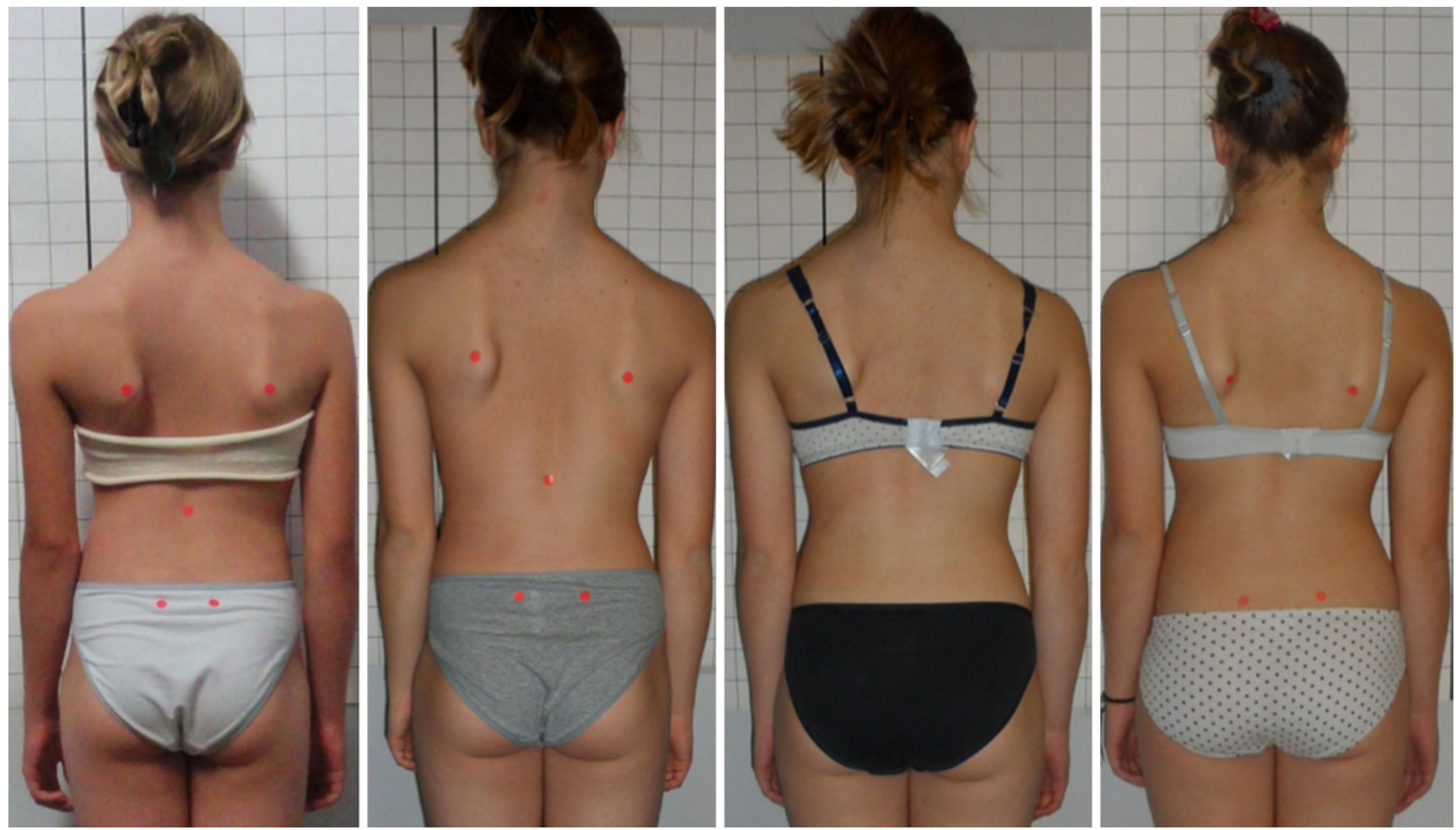

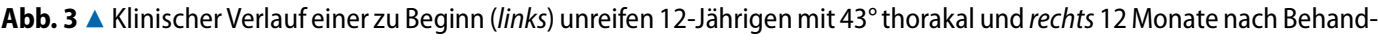
lungsabschluss deutlich kompensiert mit einer Krümmung thorakal von $35^{\circ}$
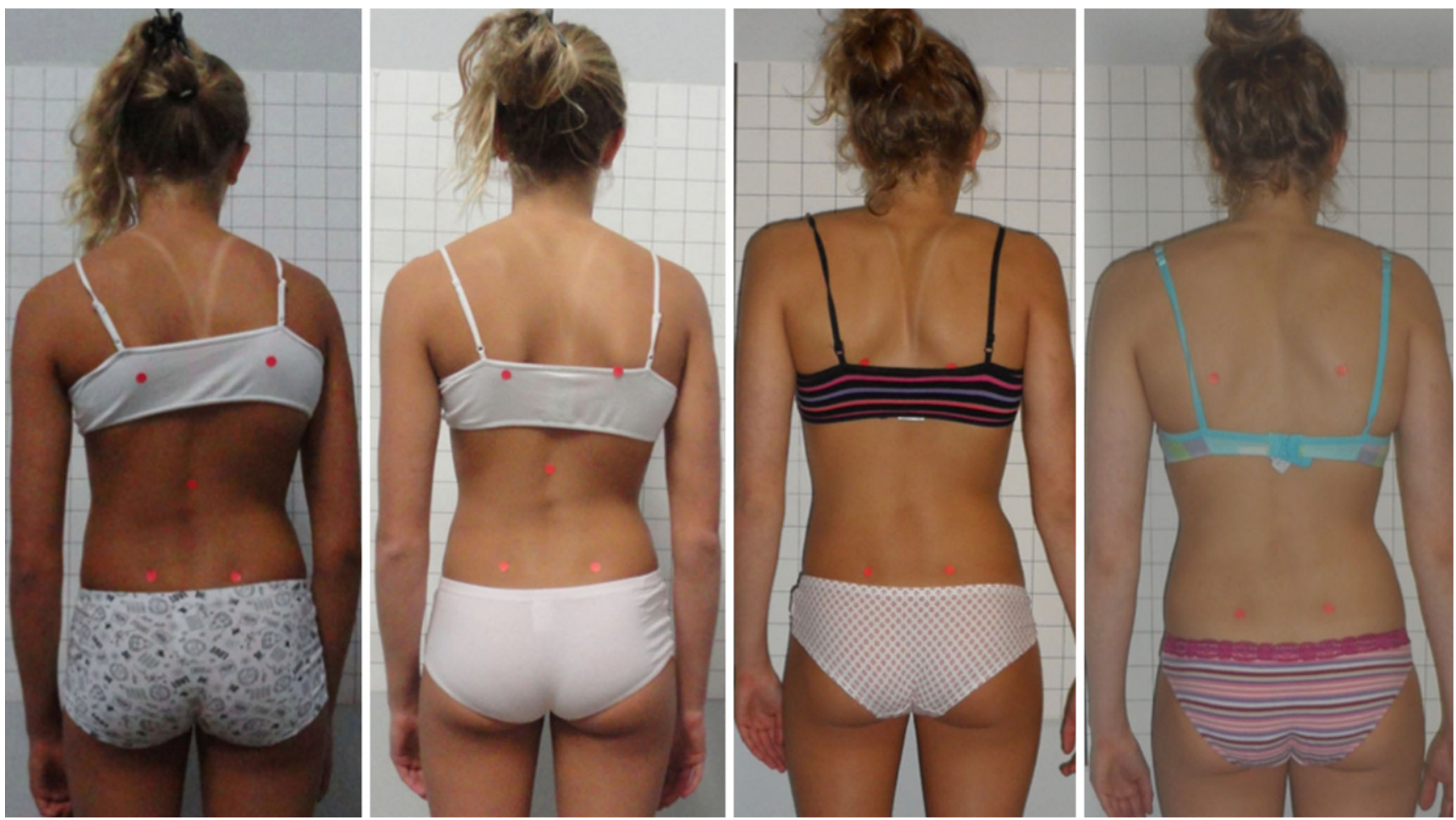

Abb. 4 ム Klinischer Verlauf einer zu Beginn (links) unreifen 13-Jährigen mit 46º thorakal, Risser 2 und rechts 6 Monate nach Behandlungsabschluss deutlich kompensiert mit einer Krümmung thorakal von $46^{\circ}$. Obwohl der Cobb-Winkel sich in diesem Fall nicht verbessert hatte, war doch eine sichtbare klinische Rekompensation zu erkennen 


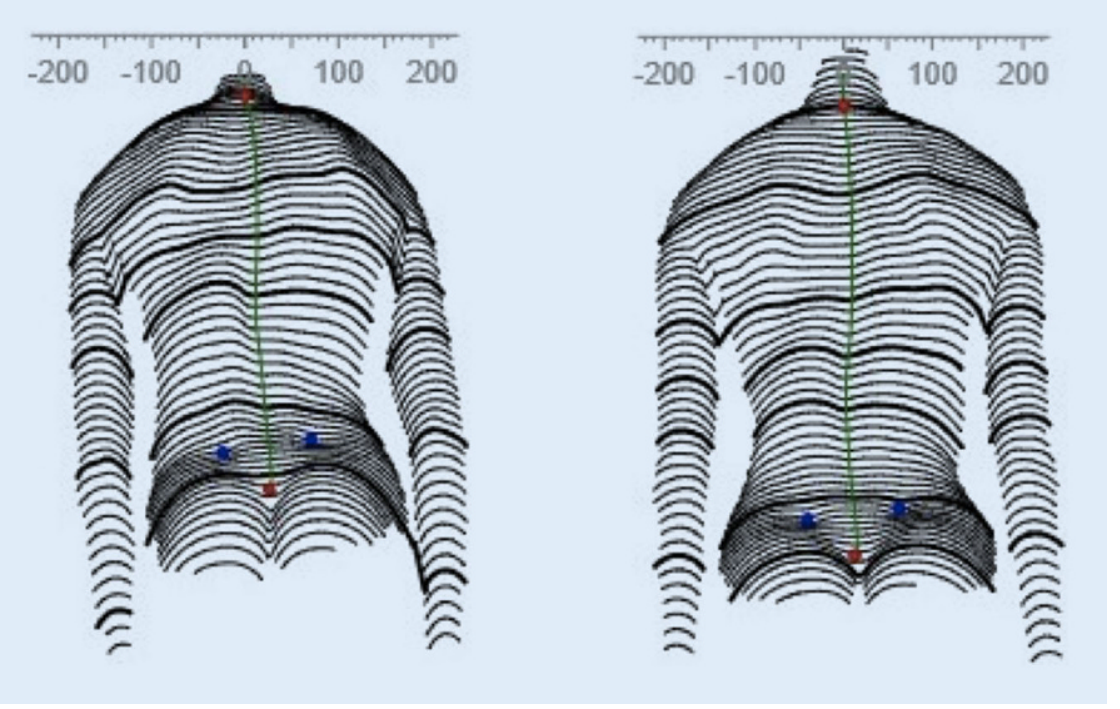

Abb. $5 \Delta$ Scan der Rückenoberfläche einer zu Beginn unreifen 13-Jährigen (links) mit $43^{\circ}$ thorakal bei einer Lenke-6-Krümmung mit Risser 2 und rechts 3 Monate nach Behandlungsabschluss deutlich kompensiert mit einer Krümmung thorakal von $37^{\circ}$. Die Profillinien haben sich nach Behandlungsabschluss deutlich abgeflacht und die klinische Verbesserung scheint deutlicher, als es bei der nur kleinen radiologischen Aufrichtung zu erwarten gewesen wäre

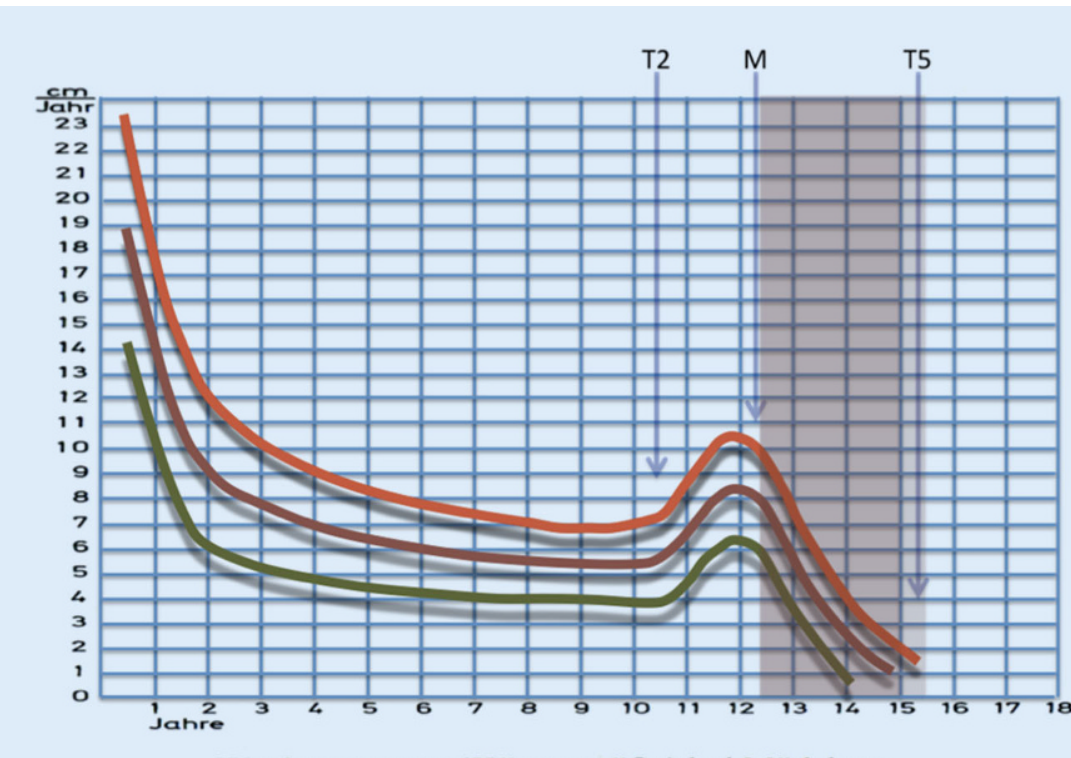

Wachstumsrate (Körpergröße) bei Mädchen.

Abb. $6 \Delta$ Die Wachstumsrate im Verlaufder Entwicklung. Tanner 2 (T2) zeigt den Beginn des puberalen Wachstumsschubes an, die Menarche $(M)$ tritt kurz nach überqueren des Wachstumsgipfels auf, eine 15-Jährige ist normalerweise nahezu ausgewachsen mit einem Tanner-Stadium von 5 (T5). Das RisserStadium 1 tritt gewöhnlich etwa 3 Monate nach Erstauftreten der Menarche ein. Der farbig unterlegte Bereich ist der Verlaufskontrollbereich der virtuellen Durchschnittspatientin unserer Gruppen (dunkel Gruppe I Verlauf von 12,3 bis 15 Jahre und hell Gruppe II von 12,3 bis 15,6 Jahre mit den Endresultaten). Die farbigen Kurven geben in etwa die Streubreite der Wachstumsdynamik wider

den sich in $\bullet$ Tab. 7, aufgeschlüsselt nach dem Risser-Stadium.

In einer aktuellen Untersuchung [27] fand sich bei Patienten mit Krümmungen zwischen 40 und $60^{\circ}$ eine Erfolgsquote von lediglich $40 \%$. Bei Berechnung des eher progredient waren als die reiferen. Einem aktuellen Review entsprechend deutet dieser Umstand eher darauf hin, dass die Korsettqualität in der genannten Studie nicht den aktuellen Möglichkeiten entsprach [42]. Die Korrektureffekte im Korsett nehmen im Allgemeinen mit zunehmender Reife ab $[3,34]$. Da eine klare Abhängigkeit des Endresultats vom erzielten Korrektureffekt im Korsett besteht $[16,26]$, sind die Endergebnisse bei qualitativ hochwertiger Korsettversorgung bei den unreiferen Patienten am besten, da deren Krümmungen am besten korrigiert werden können. Eine weitere Studie mit Patienten mit größeren Krümmungswinkeln scheint unsere Ergebnisse zu bestätigen. Auch in dieser Studie war mit qualitativ hochwertigen Korsetten eine hohe Erfolgsrate erzielt worden [3]. Diese Ergebnisse sprechen für die Notwendigkeit einer Vereinheitlichung des Versorgungsstandards mit korrigierenden Rumpforthesen für $\mathrm{Pa}$ tienten mit Skoliosen.

\section{Limitationen}

Eine Schwäche unserer Untersuchung ist die geringe Zahl der abgeschlossenen Behandlungsfälle. Leider kamen viele Patientinnen erst relativ spät mit RisserStadien von 3 und 4 in unsere Abteilung. Viele Fälle werden ja erst nach dem Hauptwachstumsschub entdeckt, in einer Phase, da der größte Teil der Progredienz bereits stattgefunden hat. Dieser Umstand spricht für die Einführung eines regelmäßigen Schoolscreenings, beginnend mit dem Auftreten der ersten Reifezeichen.

Eine weitere Schwäche unserer Untersuchung ist das Studiendesign (Chart Review). Weitere Untersuchungen mit Studiendesigns höherer Evidenz sind sicherlich sinnvoll, um die in unserer Untersuchung gefundenen Ergebnisse zu untermauern.

\section{Fazit für die Praxis}
- Gute Korrektureffekte im Korsett scheinen zu guten Endresultaten zu führen.
- Bei Ablehnung der Operation kann möglicherweise mit geeigneten Kor-




\section{setten auch jenseits der $40^{\circ}$-Grenze mit dem Aufhalten der Krümmungs- zunahme gerechnet werden. \\ - Die Behandlungssicherheit für die Pa- tienten sollte verbessert werden. Ein Ansatz hierzu kann die Verwendung standardisierter CAD(,, computer aided design")-Bibliotheken sein. - Weitere Untersuchungen mit Stu- diendesigns höherer Evidenz sind notwendig, um die in unserer Unter- suchung gefundenen Ergebnisse zu untermauern.}

\section{Korrespondenzadresse}

\section{Dr. Hans-Rudolf Weiss}

Haarbergweg 2, 55546 Neu Bamberg,

Deutschland

hr.weiss@koob-skoliose.com

\section{Einhaltung ethischer Richtlinien}

Interessenkonflikt. H.-R. Weiss hat Lizenzgebühren von der Fa. Koob GmbH \& Co KG erhalten. Zusätzlich wurden Reisekosten zu Kongressen erstattet. Die Fa. Koob GmbH \& Co KG wird von der Lebenspartnerin von H.-R. Weiss geleitet. M. Lay, S. Seibel und A. Kleban geben an, dass kein Interessenkonflikt besteht.

Für die retrospektive Studie war kein Ethikvotum notwendig. Die Vorgehensweise ist in RheinlandPfalz durch das Landeskrankenhausgesetz $\S 36, \S 37$ geregelt. Alle beschriebenen Untersuchungen am Menschen wurden mit Zustimmung der zuständigen Ethik-Kommission im Einklang mit nationalem Recht, sowie gemäß der Deklaration von Helsinki von 1975 (in der aktuellen überarbeiteten Fassung) durchgeführt. Von allen beteiligten Patienten liegt eine Einverständniserklärung vor.

Open Access. Dieser Artikel wird unter der Creative Commons Namensnennung 4.0 International Lizenz veröffentlicht, welche die Nutzung, Vervielfältigung, Bearbeitung, Verbreitung und Wiedergabe in jeglichem Medium und Format erlaubt, sofern Sie den/die ursprünglichen Autor(en) und die Quelle ordnungsgemäß nennen, einen Link zur Creative Commons Lizenz beifügen und angeben, ob Änderungen vorgenommen wurden.

Die in diesem Artikel enthaltenen Bilder und sonstiges Drittmaterial unterliegen ebenfalls der genannten Creative Commons Lizenz, sofern sich aus der Abbildungslegende nichts anderes ergibt. Sofern das betreffende Material nicht unter der genannten Creative Commons Lizenz steht und die betreffende Handlung nicht nach gesetzlichen Vorschriften erlaubt ist, ist für die oben aufgeführten Weiterverwendungen des Materials die Einwilligung des jeweiligen Rechteinhabers einzuholen.

Weitere Details zur Lizenz entnehmen Sie bitte der Lizenzinformation auf http://creativecommons.org/ licenses/by/4.0/deed.de.

\section{Literatur}

1. Asher MA, Burton DC (2006) Adolescent idiopathic scoliosis: natural history and long-term treatment effects. Scoliosis 1:2

2. Aulisa AG, Guzzanti V, Falciglia F, Galli M, Pizzetti P, Aulisa L (2017) Curve progression after long-term brace treatment in adolescent idiopathic scoliosis: comparative results between over and under 30 Cobb degrees-SOSORT 2017 award winner. Scoliosis Spinal Disord 12:36. https://doi.org/10. 1186/s13013-017-0142-y

3. Aulisa AG, Guzzanti V, Falciglia F, Giordano M, Galli M, Aulisa L (2019) Brace treatment of idiopathic scoliosis is effective for a curve over 40 degrees, but is the evaluation of Cobb angle the only parameter for the indication of treatment? Eur JPhys Rehabil Med 55(2):231-240

4. Bullmann V, Halm HF, Lerner T, Lepsien U, Hackenberg L, Liljenqvist U (2004) Prospective evaluation of braces as treatment in idiopathic scoliosis. Z Orthop Ihre Grenzgeb 142(4):403-409

5. Bunnell WP (2005) Selective screening for scoliosis. Clin Orthop Relat Res 434:40-45. https://doi.org/ 10.1097/01.blo.0000163242.92733.66

6. Chik SKT (2020) Classification and terminology. In: Moramarco M, Borysov M, Ng SY, Weiss HR (Hrsg) Schroth's textbook of scoliosis and other spinal deformities. Cambridge Scholars Publishing, Newcastle upon Tyne, S150-158

7. Daly LE, Bourke GJ (2000) Interpretation and uses of medical statistics, 5. Aufl. Blackwell Science, London, S73-74

8. Davis L, Murphy JS, Shaw KA, Cash K, Devito DP, Schmitz ML (2019) Nighttime bracing with the providence thoracolumbosacral orthosis for treatment of adolescent idiopathic scoliosis: a retrospective consecutive clinical series. Prosthet Orthot Int 43(2):158-162

9. Goldberg CJ, Moore DP, Fogarty EE, Dowling FE (2001) Adolescent idiopathic scoliosis: the effect of brace treatment on the incidence of surgery. Spine 26(1):42-47. https://doi.org/10.1097/00007632200101010-00009

10. Goldberg CJ, Moore DP, Fogarty EE, Dowling FE (2002) Adolescent idiopathic scoliosis: natural history and prognosis. Stud Health Technol Inform 91:59-63

11. Guo J, Lam TP, Wong MS et al (2014) A prospective randomized controlled study on the treatment outcome of SpineCor brace versus rigid brace for adolescent idiopathic scoliosis with follow-up according to the SRS standardized criteria. Eur Spine J 23(12):2650-2657

12. Gutman G, Benoit M, Joncas J, Beauséjour $M$, Barchi S, Labelle H et al (2016) The effectiveness of the SpineCor brace for the conservative treatment of adolescent idiopathic scoliosis. Comparison with the Boston brace. Spine J 16(5):626-631

13. Hopf C, Heine J (1985) Long-term results of the conservative treatment of scoliosis using the Chêneau brace. Z Orthop Ihre Grenzgeb 123(3):312-322

14. Korovessis P, Syrimpeis V, Tsekouras V, Vardakastanis K, Fennema P (2018) Effect of the Chêneau brace in the natural history of moderate adolescent idiopathic scoliosis in girls: cohort analysis of a selected homogenous population of 100 consecutive skeletally immature patients. Spine Deform 6(5):514-522

15. Kruzel K, Moramarco M (2020) Idiopathic scoliosis. In: Moramarco M, Borysov M, Ng SY, Weiss HR (Hrsg) Schroth's textbook of scoliosis and other spinal deformities. Cambridge Scholars Publishing Newcastle upon Tyne, S380-400

16. Landauer F, Wimmer C, Behensky H (2003) Estimating the final outcome of brace treatment for idiopathic thoracic scoliosis at 6-month followup. Pediatr Rehabil 6:201-207

17. Loder RT, Spiegel D, Gutknecht S, Kleist K, Ly T, Mehbod A (2004) The assessment of intraobserver and interobserver error in the measurement of noncongenital scoliosis in children $<$ or $=10$ years of age. Spine 29(22):2548-2553. https://doi.org/ 10.1097/01.brs.0000144828.72721.d8

18. Lonstein JE, Carlson JM (1984) The prediction of curve progression in untreated idiopathic scoliosis during growth. J Bone Joint Surg Am 66(7):1061-1071

19. Matussek J, Dingeldey E, Benditz A, Rezai G, Nahr K (2015) Conservative treatment of idiopathic scoliosis: influence of archetypical Cheneau-corsets on trunk asymmetry. Orthopäde 44(11):869-878

20. Minsk MK, Venuti KD, Daumit GL, Sponseller PD (2017) Effectiveness of the Rigo Chêneau versus boston-style orthoses for adolescent idiopathicscoliosis: a retrospective study. Scoliosis Spinal Disord 12:7

21. Misterska E, Glowacki J, Kołban M (2019) Does rigid spinal orthosis carry more psychosocial implications than the flexible brace in AIS patients? A cross-sectional study. J Back Musculoskelet Rehabil 32(1):101-109. https://doi.org/10.3233/ BMR-181121

22. Murrell GA, Coonrad RW, Moorman CT 3rd, Fitch RD (1993) An assessment of the reliability of the Scoliometer. Spine 18(6):709-712.https://doi.org/ 10.1097/00007632-199305000-00006

23. Nachemson AL, Peterson LE (1995) Effectiveness of treatment with a brace in girls who have adolescent idiopathic scoliosis. A prospective, controlled study based on data from the brace study of the scoliosis research society. J Bone Joint Surg Am 77(6):815-822

24. Pham VM, Herbaux B, Schill A, Thevenon A (2007) Evaluation of the Cheneau brace in adolescent idiopathic scoliosis. Ann Readapt Med Phys 50(3):125-133

25. Richards BS, Bernstein RM, D'Amato CR, Thompson GH (2005) Standardization of criteria for adolescent idiopathic scoliosis brace studies: SRS committee on bracing and nonoperative management. Spine 30(18):2068-2077. https://doi.org/ 10.1097/01.brs.0000178819.90239.d0

26. van den Bogaart $M$, van Royen $B J$, Haanstra $T M$, de Kleuver M, Faraj SSA (2019) Predictive factors for brace treatment outcome in adolescent idiopathic scoliosis: a best-evidence synthesis. Eur Spine J 28(3):511-525. https://doi.org/10.1007/s00586018-05870-6

27. Verhofste BP, Whitaker AT, Glotzbecker MP et al (2020) Efficacy of bracing in skeletally immature patients with moderate-severe idiopathicscoliosis curves between $40^{\circ}$ and 60 . Spine Deform. https:// doi.org/10.1007/s43390-020-00131-3

28. Wang J, Zhang J, Xu R, Chen TG, Zhou KS, Zhang HH (2018) Measurement of scoliosis Cobb angle by end vertebra tilt angle method. J Orthop Surg Res 13(1):223. https://doi.org/10.1186/s13018-0180928-5

29. Weinstein SL, Dolan LA, Spratt KF, Peterson KK, Spoonamore MJ, Ponseti IV (2003) Health and function of patients with untreated idiopathic scoliosis: a 50-year natural history study. JAMA 289(5):559-567 
30. WeinsteinSL,DolanLA,WrightJG,DobbsMB(2013) Effects of bracing in adolescents with idiopathic scoliosis. NEngl J Med 369(16):1512-1521

31. Weiss HR, Lehnert-Schroth C (1995) Breathing excursions in scoliosis patients. In: D'Amico M, Me rolli A, Santambrogio GC (Hrsg) Threedimensional analysis of spinal deformities. Studies in health technology and Informatics, Bd. 15. IOS Press, Amsterdam, S285-288

32. Weiss HR, Weiss GM (2005) Brace treatment during pubertal growth spurt in girls with idiopathic scoliosis (IS): a prospective trial comparing two different concepts. Pediatr Rehabil 8(3):199-206

33. Weiss $H$, Negrini $S$, Rigo M et al (2006) Indications for conservative management of scoliosis (guidelines). Scoliosis 1:5

34. Weiss HR, Werkmann M, Stephan C (2007) Correction effects of the ScoliOlogiC "Chêneau light" brace in patients with scoliosis. Scoliosis 2:2 https://doi.org/10.1186/1748-7161-2-2

35. Weiss HR (2010) "Brace technology" thematic series - the Gensingen brace ${ }^{\mathrm{TM}}$ in the treatment of scoliosis. Scoliosis 5:22. https://doi.org/10.1186/ 1748-7161-5-22

36. Weiss HR, Moramarco M (2013) Remodelling of trunk and backshape deformities in patients with scoliosis using standardized asymmetric computer-aided design/computer-aided manufacturing braces. Hard Tissue 2(2):14

37. Weiss HR, Kleban A (2015) Development of CAD/CAM based brace models for the treatment of patients with scoliosis-classification based approach versus finite element modelling. Asian Spine J9(5):661-667

38. Weiss HR, Karavidas N, Moramarco M, Moramarco K (2016) Long-term effects of untreated adolescent idiopathic scoliosis: a review of the literature. Asian Spine J 10(6):1163-1169

39. Weiss HR, Tournavitis T, Nan XF, Borysov M, Paul L (2017) Workflow of CAD / CAM scoliosis brace adjustment in preparation using 3D printing. Open Med Inform J 11:44-51

40. Weiss HR, Turnbull D, Seibel S, Kleban A (2019) First end-result of a prospective cohort with AIS treated with a CAD Chêneau style brace. J Phys Ther Sci 31(12):983-991

41. Weiss HR, Turnbull D (2020) Best practice recommendations for the conservative treatment of patients with spinal deformities. In: Moramarco M, Borysov M, Ng SY, Weiss HR (Hrsg) Schroth's textbook of scoliosis and other spinal deformities. Cambridge Scholars Publishing, Newcastle upon Tyne, S760-775

42. Weiss HR, Turnbull D (2020) Brace treatment for children and adolescents with scoliosis. IntechOpen. https://doi.org/10.5772/intechopen. 91234

43. Wong MS, Cheng JC, Lam TP et al (2008) The effect of rigid versus flexible spinal orthosis on the clinical efficacy and acceptance of the patients with adolescent idiopathic scoliosis. Spine 33(12):1360-1365

44. Zaborowska-Sapeta K, Kowalski IM, Kotwicki T, Protasiewicz-Faldowska H, Kiebzak W (2011) Effectiveness of Cheneau brace treatment for idiopathic scoliosis: prospective study in 79 patients followed to skeletal maturity. Scoliosis 6(1):2

\section{Neue G-BA Richtlinie zum Umgang mit gerinnungshemmender Medikation bei Versorgung der hüftgelenknahen Femurfraktur in Kraft getreten}

Kurzfassung

Der Gemeinsame Bundesausschusses (G-BA) nimmt in seiner neuesten Veröffentlichung Stellung zum Umgang mit Antikoagulantien bei der Akutversorgung hüftgelenksnaher Femurfrakturen, insbesondere zu den DOAKs. Ein zentrales Thema ist dabei der Nachweis von DOAKs mit spezifischen Point-of-Care Tests. Hierin wird auf den DOAC Dipstick (DOASENSE) als für die Entscheidungsfindung geeigneten Vor-Ort-Test in Notfallsituationen verwiesen.

\section{Problematik}

Eine Zeitverzögerung bei der operativen Versorgung von Patienten mit hüftgelenksnahen Femurfrakturen geht mit erhöhter Morbidität und Mortalität einher. Daher sind alle Kliniken bemüht, diese Patienten schnellstmöglich zu operieren. Inzwischen nehmen mehr als 50\% der Patienten mit Vorhofflimmern ein DOAK zur Antikoagulation ein. Dies erfordert zur Frage der Operationsfähigkeit im Hinblick auf potentielle Blutungskomplikationen eine schnelle und zuverlässige Abklärung durch einen POC Test.

Aufgrund der Unsicherheit der anamnestischen Angaben kommt es mit den bekannten spezifischen Gerinnungsmethoden hinsichtlich der DOAK-Einnahme regelmäßig zu Zeitverzögerungen. Viele Kliniken die in Deutschland hüftgelenksnahe Frakturen operieren, sind nicht in der Lage eine schnelle und spezifische Gerinnungsdiagnostik auf DOAKs durchzuführen. Die meisten Labore halten lediglich Gerinnungsglobaltests wie PT und aPTT vor, die aber für DOAKs keine valide Aussage zulassen $(1,2)$.

\section{Der Beschluß}

In dem Beschluß (3) präzisierte der G-BA die SOP zum Umgang mit gerinnungshemmender Medikation. Liegt bei Patienten, die einen Gerinnungshemmer einnehmen, keine verlässliche Angabe zum letzten Einnahmezeitpunkt vor, müssen Kliniken innerhalb von $24 \mathrm{~h}$ eine zusätzliche Einschätzung des $\mathrm{Ge}-$ rinnungsstatus mit geeigneten Testverfahren auch für DOAKs durchführen.

Aufgrund der begrenzten Laborausstattung vieler Kliniken kann dieser Beschluß diese
Kliniken in zeitliche Bedrängnis bringen. Eine mögliche Lösung des Problems wird in den Tragenden Gründen (3) zum Beschluß genannt. Hier wird auf den seit einiger Zeit in Deutschland und international erhältlichen CE-zertifizierten Point-of Care Test der Firma DOASENSE (4) verwiesen: „Für alle vier gängigen DOAKs ist auch ein Urin-Dipstick-Test mit Farbkodierung vorhanden, der mit sehr hoher Zuverlässigkeit anzeigt, ob relevante Wirkstoffkonzentrationen im Urin vorhanden sind." und „Die Testgenauigkeit in der Studie war hoch (Sensitivität und Spezifität jeweils $>95 \%)$.... Damit ist der Test grundsätzlich als Vor-Ort-Test in Notfallsituationen geeignet".

Dieser Test ist mittlerweile in ca. 100 Kliniken in Deutschland eingeführt und kann direkt von DOASENSE (4) oder dem Vertriebspartner Hitado (5) bezogen werden.

1.) Gosselin RC https://doi.org/10.1055/s0038-1627480
2.) Gosselin RC 33-39.DOI:
10.1111/ijlh.12992
3.) www.g-ba.de/beschluesse/4655/
4.) www.doasense.de
5.) www.hitado.de 